\title{
Plasma Temperature Determination of Hydrogen Containing High-Frequency Electrodeless Lamps by Intensity Distribution Measurements of Hydrogen Molecular Band
}

\author{
Zanda Gavare, Gita Revalde, and Atis Skudra \\ Institute of Atomic Physics and Spectroscopy, University of Latvia, 4 Skunu Street., Riga LV-1050, Latvia \\ Correspondence should be addressed to Zanda Gavare, zanda.gavare@gmail.com
}

Received 15 April 2009; Revised 29 July 2009; Accepted 23 September 2009

Academic Editor: Chantal Stehle

Copyright ( $) 2010$ Zanda Gavare et al. This is an open access article distributed under the Creative Commons Attribution License, which permits unrestricted use, distribution, and reproduction in any medium, provided the original work is properly cited.

\begin{abstract}
The goal of the present work was the investigation of the possibility to use intensity distribution of the Q-branch lines of the hydrogen Fulcher- $\alpha$ diagonal band $\left(d^{3} \Pi_{u}^{-} \rightarrow a^{3} \sum_{g}^{+}\right.$electronic transition; Q-branch with $\left.v=v^{\prime}=2\right)$ to determine the temperature of hydrogen containing high-frequency electrodeless lamps (HFEDLs). The values of the rotational temperatures have been obtained from the relative intensity distributions for hydrogen-helium and hydrogen-argon HFEDLs depending on the applied current. The results have been compared with the method of temperature derivation from Doppler profiles of He $667.8 \mathrm{~nm}$ and Ar $772.4 \mathrm{~nm}$ lines. The results of both methods are in good agreement, showing that the method of gas temperature determination from the intensity distribution in the hydrogen Fulcher- $\alpha$ (2-2)Q band can be used for the hydrogen containing HFEDLs. It was observed that the admixture of $10 \%$ hydrogen in the argon HFEDLs significantly reduces the gas temperature.
\end{abstract}

\section{Introduction}

The high-frequency electrodeless lamps (HFEDLs) are well known as a bright radiators of narrow and intense spectral lines in wide spectral range from vacuum ultra violet to infrared. These lamps are widely used in Atomic Absorption Spectroscopy for the determination of metal concentrations $[1,2]$, and they are of interest in plasma-surface interaction investigations due to lack of electrodes [3, 4]. For the application of HFEDLs and for the discharge plasma modeling it is important to estimate the plasma parameters in the lamps. It is of particular importance to determine and control the gas temperature, since this parameter plays an important role for many relevant plasma processes. In our previous work for the discharge gas temperature estimation in HFEDLs we used the high-resolution spectroscopy method of the emission spectral line shape measurements using Fabry-Perot interferometer and Zeeman spectrometer and nonlinear spectral line shape modeling [5]. However, the method of the line shape modeling is very complicated due to the necessity to solve the incorrect inverse task [6]. It is of great interest to find other methods and verify their applicability for the determination of plasma temperature in HFEDLs. In the case of hydrogen containing plasmas, one of the commonly used techniques for the determination of gas temperature is based on the measurements of the intensity distribution in the rotational bands of hydrogen molecule [7-18], for instance, (0-0), (11), (2-2), and (3-3) Q-branches of Fulcher- $\alpha\left(d^{3} \Pi_{u}^{-} \rightarrow\right.$ $a^{3} \Sigma_{g}^{+}$) electronic transition [9-18]. The determination of the gas temperature from measured intensity distribution in rotational bands is based on a certain theoretical model, which involves series of assumptions about the mechanism of the excitation-deexcitation of rotational-vibrational (vibronic) levels of hydrogen. According to this model, the translational gas temperature may be in equilibrium with rotational temperature determined from either upper or lower energy level depending on processes in plasma under study $[8,12,14]$.

For example, as it was shown by Tomasini et al. [12], in the case of hydrogen containing microwave discharge the ground state rotational temperature can be used at pressure of 0.5 Torr to derive the gas temperature, while at higher pressure (1 Torr) the authors observed discrepancy between temperature obtained from the Doppler broadening 
and rotational temperature derived from the ground state energies.

Garg et al. [14] compared temperature values obtained from hydrogen Fulcher- $\alpha(0-0)$ Q-branch with those derived from the rotational band of $\mathrm{N}_{2}$ molecule. They found that in the microwave plasma at pressure 10 Torr, the $\mathrm{H}_{2}$ rotational temperatures derived using upper and lower level rotational constants are far from equilibrium with translational temperature and they do not accurately represent the translational temperature under their experimental conditions.

Iordanova [17] used the ground state rotational temperature of hydrogen molecule to derive temperature of the RF (excited at $27 \mathrm{MHz}$ frequency) inductively driven $\mathrm{H}_{2}$ plasma in the pressure range $26-60$ mTorr, estimating that in this pressure range and at gas temperatures $300-900 \mathrm{~K}$ the characteristic time between heavy particle collisions is much longer than the radiation lifetime of the excited state, which means that the rotational distribution in the excited state is an image of the rotational distribution in the ground state.

Lebedev and Mokeev [18] used Fulcher- $\alpha$ (2-2)Q-branch to determine the microwave plasma temperature at pressures 1-8 Torr. They argued that at pressure 1 Torr the collision frequency $v_{\text {coll }}$ is much smaller than the radiative destruction frequency $v^{*}\left(v_{\text {coll }} \ll v^{*}\right)$, concluding that under their experimental conditions the gas temperature can be derived from the ground state rotational energies.

In each particular case, the relation between the rotational and translational temperatures needs to be verified. One way to establish the relationship between both temperatures is the comparison of the rotational temperature with that obtained by other methods, for example, the rovibrational bands of $\mathrm{N}_{2}$ molecule [14] or Doppler broadening of the lines [12].

The Fulcher- $\alpha$ Q-branches have been used for the plasma temperature determination in different kinds of discharges; however for the diagnostics of the high-frequency electrodeless discharge lamps (excited at about $100 \mathrm{MHz}$ frequency) it was not used before. Therefore, the goal of the present work was to investigate the possibility to use relative intensities of the Q-branch lines of the hydrogen Fulcher- $\alpha$ diagonal band $\left(d^{3} \Pi_{u}^{-} \rightarrow a^{3} \Sigma_{g}^{+}\right.$electronic transition; Q branch with $v=$ $v^{\prime}=2$ ) in spectroscopic diagnostics of hydrogen containing HFEDLs. In this paper, the $\mathrm{H}_{2}$ rotational temperature was obtained from the intensity measurements of Fulcher- $\alpha$ (2-2) Q-branch, and the results were compared with the temperature derived from the measurements of Doppler broadening of $\mathrm{Ar}$ and $\mathrm{He}$ atomic lines.

\section{Experiment}

The plasma sources under study was helium HFEDLs with hydrogen $\left(\mathrm{p}_{\mathrm{He}} \approx 0.9\right.$ Torr, $\mathrm{p}_{\mathrm{H} 2} \approx 0.1$ Torr $)$ and argon HFEDLs with hydrogen $\left(\mathrm{p}_{\mathrm{Ar}} \approx 0.9\right.$ Torr, $\mathrm{p}_{\mathrm{H} 2} \approx$ 0.1 Torr) manufactured at the Institute of Atomic Physics and Spectroscopy, University of Latvia. The cylindrical lamps with diameter of $2 \mathrm{~cm}$ and the length of $4 \mathrm{~cm}$ were placed into an induction coil and an inductive coupled discharge was exited by means of a high-frequency field of about $100 \mathrm{MHz}$ frequency. The power of the discharge was changed, changing the applied current $i$ into the coil in the region 80-200 mA. The gas temperature dependence from the current $i$ in the induction coil was investigated.

The gas temperature of hydrogen and helium or argon containing HFEDL has been measured by two methods: using the relative intensities of the hydrogen (2-2)Q branch of Fulcher- $\alpha$ band and using the temperature derivation from Doppler profile of $\mathrm{He} 667.8 \mathrm{~nm}\left(\mathrm{He}\right.$ I $\left.2{ }^{1} \mathrm{P}-3{ }^{1} \mathrm{D}\right)$ and $\mathrm{Ar}$ $772.4 \mathrm{~nm}\left(\right.$ Ar I $\left.1 s_{5}-2 \mathrm{p}_{7}\right)$ lines.

\subsection{Experimental Setup for the Rotational Temperature} Determination. The light from the lamp was imaged on the entrance slit of the spectrometer (JobinYvon SPEX $1000 \mathrm{M}$, grating $1200 \mathrm{l} \cdot \mathrm{mm}^{-1}$, focal length $1 \mathrm{~m}$ ) and detected by means of a charge-coupled device matrix detector $(2048 \times$ 512 Thermoelectric Front Illuminated UV Sensitive CCD Detector, Simphony). With this detector a spectral range of about $15 \mathrm{~nm}$ can be recorded at one time. The resolving power of the system spectrometer-CCD camera was $\sim 136$ pixels $\cdot \mathrm{nm}^{-1}$. The entrance slit was set at $30 \mu \mathrm{m}$. In Figure 1 the experimental setup for the temperature measurements is shown.

The emission spectra of the discharge under study have been recorded in the wavelength range $\lambda=620-630 \mathrm{~nm}$, containing first five lines of the (2-2)Q branch of the Fulcher$\alpha$ band system of the hydrogen molecule. Figure 2 shows an example of the recorded emission spectra of hydrogen containing HFEDL.

2.2. Experimental Setup for the Gas Temperature Determination from the Line Profile. The experimental setup for the spectral line shape registration by means of the FabryPerot interferometer is shown in Figure 3. The light, collected from the lamp, is transmitted through the pressure-scanned interferometer, focused on a monochromator, amplified and registered by means of a photomultiplier. Line profiles of $\mathrm{He}$ $667.8 \mathrm{~nm}$ (or Ar $772.4 \mathrm{~nm}$ ) line were recorded using mirrors with a dielectric coating and a $1.4 \mathrm{~cm}$ spacer (free spectral range of $0.36 \mathrm{~cm}^{-1}$ ).

The experimental work was organised in the following way. First, the measurement session was performed to obtain the gas temperature from the Fulcher- $\alpha$ band system of hydrogen (the first session). The first five lines $\mathrm{Q}_{1}-\mathrm{Q}_{5}$ could be distinguished and their intensity measured. The second session of measurements was performed to derive the temperature from the Doppler profile of helium or argon lines. After the second measurement session the control measurements of the lamp spectra was made (the control session).

\section{Theoretical}

3.1. Rotational Temperature Determination. The determination of the rotational temperature is based on the measurements of relative intensity distributions in the vibronic bands of Fulcher- $\alpha$ system; in this case, rovibronic line $\left(\mathrm{Q}_{1}-\mathrm{Q}_{5}\right)$ intensities of Q-branch of the hydrogen Fulcher- $\alpha$ diagonal band $\left(d^{3} \Pi_{u}^{-} \quad(v=2) \rightarrow a^{3} \Sigma_{g}^{+} \quad\left(v^{\prime}=2\right)\right.$ electronic transition $)$ 


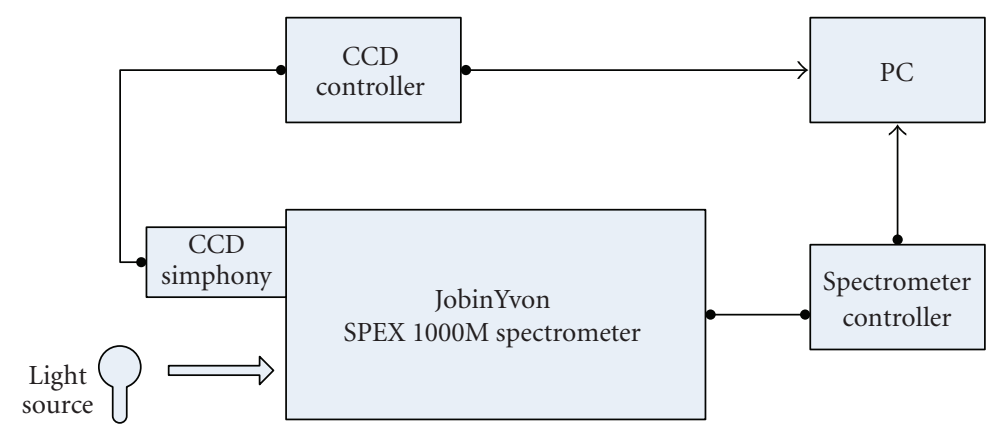

FIGURE 1: The experimental setup for the measurements of gas temperature using $\mathrm{H}_{2}$ Fulcher- $\alpha$ (2-2)Q band.

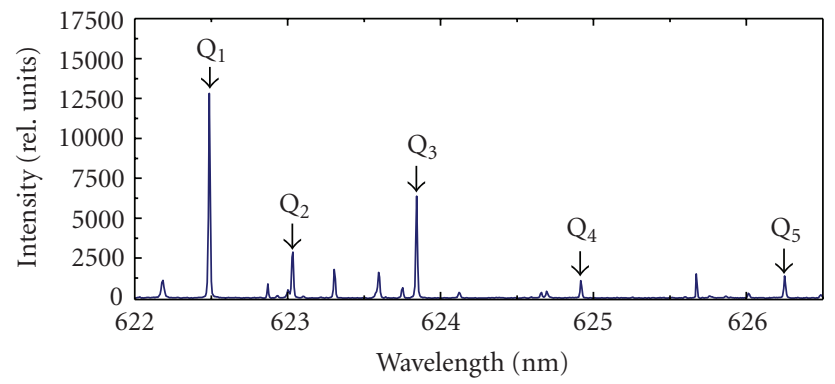

Figure 2: Example of a typical emission spectra of the hydrogen containing plasma of HFEDL recorded at applied current $i=$ $80 \mathrm{~mA}$, in the wavelength region containing five lines of the (2-2)Q branch of the Fulcher- $\alpha$ band system of the $\mathrm{H}_{2}$ molecule.

have been used. The transition diagram for these lines is shown in Figure 4.

The method of the determination of the gas temperature is described in detail in $[8-10,16,19-21]$.

According to this model, the translational gas temperature may be in equilibrium with rotational temperature determined from either upper or lower energy level depending on processes in plasma under study $[8,12,14,16]$.

(i) The rotational temperature determined from the excited upper level can be equal to the translational temperature if the rotational relaxation time from the upper to the lower level is much smaller than the radiative lifetime (the destruction frequency of the $d^{3} \Pi_{u}$ state $v^{*}$ is lower than the neutral species rotational mixing collision frequency $\left.v_{\text {coll }}, v^{*}<v_{\text {coll }}\right)$, and equilibrium between the rotational distribution and the neutral velocity distribution is achieved.

(ii) If the upper level is only populated by direct electron impact from the ground state, then the upper level rotational distribution is the image of the lower level rotational distribution. In this case the rotational temperature obtained from the lower level can be considered as a valid estimation of translational temperature. This condition is satisfied for lowpressure plasmas $[14,17]$.

It is necessary to mention that for the precise temperature derivation from the Fulcher- $\alpha$ bands it is necessary to take

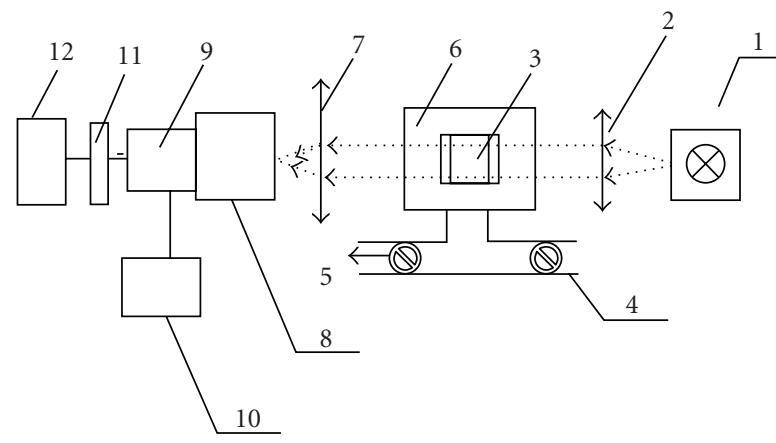

FIGURE 3: Experimental setup for spectral line profile measurements based on a pressure scanned Fabry-Perot interferometer. 1: Lamp inside the generator; 2: lens; 3: Fabry-Perot interferometer; 4, 5: capillary; 6: vacuum chamber; 7: lens; 8: monochromator; 9: photomultiplier; 10: power supply; 11: amplifier; 12: PC.

into account the excitation rate and vibrational distribution, for example, as it was done in [19-21]. Kado et al. in their work [19] show that there is a small discrepancy between values of rotational temperature derived with and without taking into account the vibrational excitation. However within the framework of this study we will use simple model with the following assumptions $[8-10,16]$ :

(1) the population distribution in the ground $X^{1} \Sigma_{g}^{+}$ $\left(v^{\prime \prime}=0\right)$ vibronic state obeys Boltzmann's law (with the rotational temperature equal to gas temperature $T_{g}$ );

(2) the excited states are populated mainly via electron collisions from the ground $X^{1} \Sigma_{g}^{+}\left(v^{\prime \prime}=0\right)$ vibronic state;

(3) the transitions with a change in angular momentum $|\Delta N| \geq 2$ may be neglected, and the rate coefficients are assumed to be independent of the rotational quantum number;

(4) the effective lifetime of the excited state does not depend on the rotational quantum number and is much shorter than the relaxation time of rotational levels.

In addition to the previous assumptions, if we assume that the rovibronic transition probability of the Q lines of 


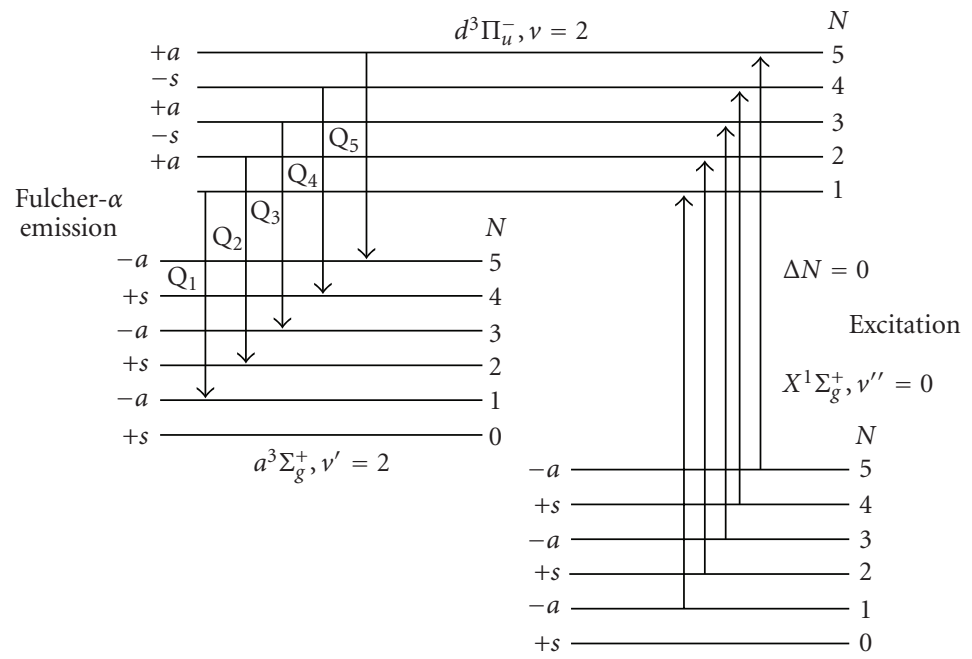

Figure 4: The transition diagram of the first five lines of hydrogen Fulcher- $\alpha$ (2-2)Q band.

TABle 1: Transition parameters for the first five lines of (2-2)Q branch of Fulcher- $\alpha$ band of hydrogen molecule [22-24].

\begin{tabular}{lcccc}
\hline Line & $E_{X 0 N}, K$ & $N$ & $g_{a, s}$ & $\lambda, \mathrm{nm}$ \\
\hline $\mathrm{Q}_{1}$ & 170,50 & 1 & 3 & 622.4815 \\
$\mathrm{Q}_{2}$ & 509,80 & 2 & 1 & 623.0258 \\
$\mathrm{Q}_{3}$ & 1015,10 & 3 & 3 & 623.8391 \\
$\mathrm{Q}_{4}$ & 1681,60 & 4 & 1 & 624.9150 \\
$\mathrm{Q}_{5}$ & 2503,80 & 5 & 3 & 626.2495 \\
\hline
\end{tabular}

Fulcher- $\alpha$ (2-2) band does not depend on the rotational quantum number, the population of the level $d^{3} \Pi_{u}^{-}(v=2$, $N)$ is related to the rotational temperature in the following way:

$$
\begin{aligned}
\frac{N_{n v N}}{g_{a, s} \cdot(2 N+1)} & \propto \frac{I_{n^{\prime} v^{\prime} N^{\prime}}^{n v N}}{\left(v_{n^{\prime} v^{\prime} N^{\prime}}^{n v N}\right)^{3} \cdot g_{a, s} \cdot(2 N+1)} \\
& \propto \exp \left(-\frac{E_{X 0 N}}{T_{\text {rot }}}\right),
\end{aligned}
$$

where $N_{n v N}$ is the population of the level $d^{3} \Pi_{u}^{-}(v=2$, $N), I_{n^{\prime} v^{\prime} N^{\prime}}^{n v N}$ is measured intensity of a spectral line, $v_{n^{\prime} v^{\prime} N^{\prime}}^{n v N}$ is wavenumber of the radiative transition (in $\mathrm{cm}^{-1}$ ), $g_{a, s}$ is statistical weight of the $d^{3} \Pi_{u}^{-}(v=2, N)$ level, $N$ is rotational quantum number, $E_{X 0 N}$ is rotational energy of the ground state (in K), and $T_{\text {rot }}$ is the temperature (in K). From (1) one may conclude that it is possible to determine the rotational temperature (equal to the gas temperature) by measuring the line intensities and knowing the transition parameters. The transition parameters for the lines of (2-2)Q branch of Fulcher- $\alpha$ band can be seen in Table 1 [22-24].

To determine the plasma temperature the formula (1) can be expressed through the logarithm in the following way:

$$
\ln \left(\frac{I_{n^{\prime} v^{\prime} N^{\prime}}^{n v N}}{\left(v_{n^{\prime} v^{\prime} N^{\prime}}^{n v N}\right)^{3} \cdot g_{a, s} \cdot(2 N+1)}\right)=-\frac{E_{X 0 N}}{T_{\text {rot }}}+\text { const, }
$$

where in const all factors, which do not depend on the rotational quantum number, are combined. For the determination of the gas temperature it is necessary to plot the dependence of the logarithm of the reduced line intensity (left side of (2)) on the molecular rotational energy in the ground state $E_{X 0 N}$. A typical semilogarithmic plot of the Fulcher- $\alpha(2-2) \mathrm{Q}$ branch lines is shown in Figures 5(a) and 5(b) for hydrogen-helium and hydrogen-argon HFEDLs, respectively. The temperature then can be obtained by applying the linear fit.

The linearity of these plots (Figure 5) indicates the Boltzmann distribution over the rotational levels. It can be seen that the point corresponding to the line $\mathrm{Q}_{5}$ is higher (especially for the $\mathrm{Ar}+\mathrm{H}_{2} \mathrm{HFEDL}$, see Figure 5(b)), indicating the deviation from the Boltzmann distribution for this rotational level. The difference is only about 5\%; yet it was excluded from the plots of $\mathrm{Ar}+\mathrm{H}_{2}$ HFEDL.

3.2. Gas Temperature Determination from the Line Profile. The gas temperature of the plasma under study has been obtained from the Doppler widths of the shapes of spectral lines (He $667.8 \mathrm{~nm}$ or Ar $772.4 \mathrm{~nm}$ ), measured using FabryPerot interferometer.

The problem is that spectral line profile, registered by means of the interferometer, differs significantly from the real one. The observed distribution $f(x)$ is given by a convolution:

$$
f(x)=\int_{-\infty}^{+\infty} f^{\prime \prime}(x-y) \cdot f^{\prime}(y) d y+\xi(x)
$$

where $f^{\prime \prime}(x)$ is the real profile of a spectral line, $f^{\prime}(x)$ is the instrument function, and $\xi(x)$ is the function characterizing random errors. To determine the real spectral line profile $f^{\prime \prime}(x)$ (in our case-the real Doppler profile), it is necessary to solve the inverse task (see (3)). We used another methodthe line fitting by means of a nonlinear multiparameter chisquare fit. It was assumed that the form of the experimental 


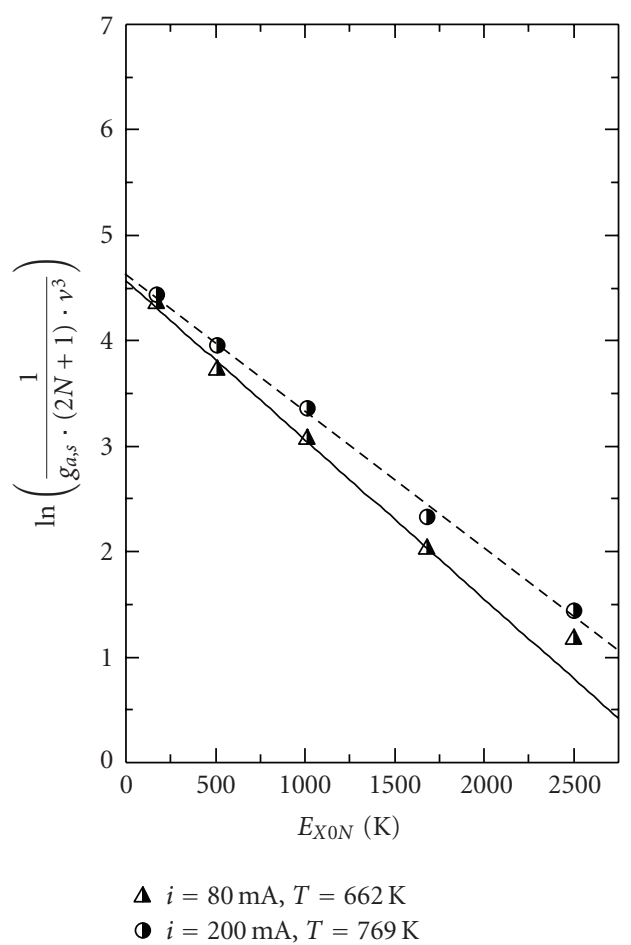

(a)

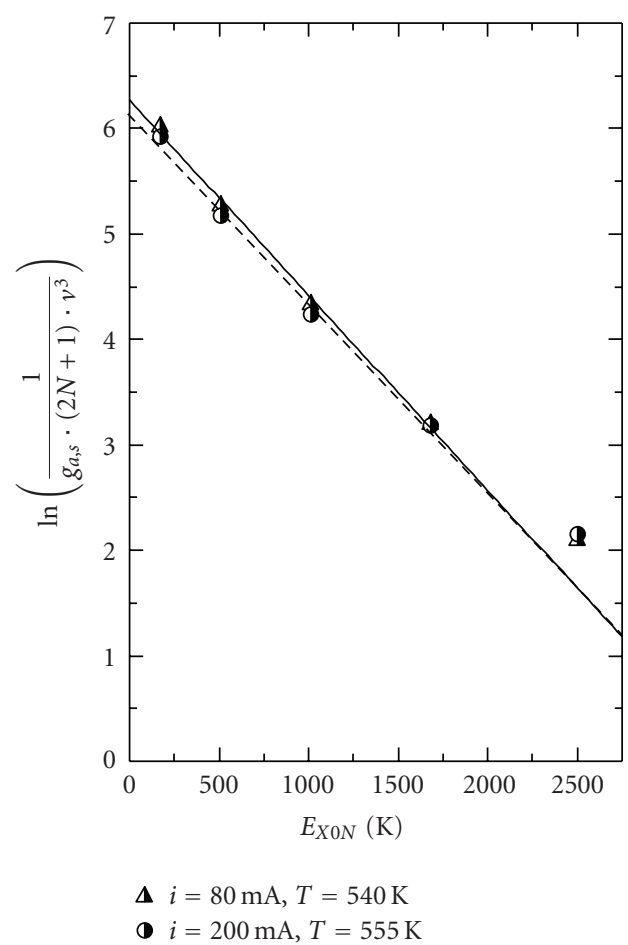

(b)

FIGURE 5: The semilogarithmic plot of scaled intensity of Fulcher-a (2-2)Q lines versus rotational energy of the molecular hydrogen ground state: (a) $\mathrm{He}+\mathrm{H}_{2}$ HFEDL and (b) $\mathrm{Ar}+\mathrm{H}_{2} \mathrm{HFEDL}$, at $i=80 \mathrm{~mA}$ and $i=200 \mathrm{~mA}$.

profile can be approximated by the Voigt function, which is a convolution of the Gaussian and Lorentz functions:

$$
\begin{gathered}
V(a, \omega)=\frac{a}{\pi} \int_{-\infty}^{+\infty} \frac{\exp \left(-z^{2}\right) d z}{a^{2}+(\omega-y)^{2}}, \\
a=\frac{\Delta \nu_{L}}{\Delta \nu_{G}} \sqrt{\ln 2}, \quad \omega=\frac{2\left(\nu-\nu_{0}\right)}{\Delta \nu_{G}} \sqrt{\ln 2}, \\
z=\frac{\left(\nu-\nu^{\prime}\right)}{\Delta \nu_{G}} \sqrt{\ln 2},
\end{gathered}
$$

where $V(a, \omega)$ is Voigt function describing the real line profile, $\Delta v_{L}$ is the Lorentzian width, $\Delta v_{G}$ is the Gaussian full-width at half-maximum (FWHM), $\nu_{0}$ is line-center frequency, and $v$ is frequency. The profiles were fitted and deconvoluted from the Lorentz function, mainly composed of the instrumental function in our case, to obtain the real Doppler profile. In the case of helium and argon discharges we can neglect the effect of self-absorption which often has to be taken into account [5]. An example of experimental profile of He $667.8 \mathrm{~nm}$ line is shown in Figure 6.

The gas temperature has been calculated by well-known formula:

$$
T=\mu \cdot\left(\frac{\Delta \lambda_{D}}{\lambda_{0}} \cdot \frac{1}{7,16 \cdot 10^{-7}}\right)^{2},
$$

where $T$ is gas temperature $(\mathrm{K}), \Delta \lambda_{D}$ is Doppler width (FWHM), $\lambda_{0}$ is wavelength at the centre of the line, and $\mu$ is atomic mass of the species (in this case-atomic mass of helium or argon).

\section{Results and Discussion}

4.1. He and $\mathrm{H}_{2}$ HFEDLs. In Figure 7 one may see the comparison of the temperature for the $\mathrm{He}$ and $\mathrm{H}_{2}$ HFEDL, obtained by means of the two methods: using the intensities of the $\mathrm{H}_{2}$ rotational lines of the (2-2)Q branch of the Fulcher$\alpha$ band system and using the Doppler broadening of $\mathrm{He}$ $667.8 \mathrm{~nm}$ line.

The gas temperature ranging from $630 \mathrm{~K}$ to $740 \mathrm{~K}$ was obtained, depending on the applied current (80-200 mA). Relative uncertainty of the obtained temperature values using both methods is less than $10 \%$ (the uncertainties were obtained averaging results from the repeated measurements, the dispersion is due to the variances in the line intensities from measurement to measurement), and the difference between the results of both methods does not exceed $10 \%$, too.

These results show that in the case of hydrogen containing helium-HFEDL the rotational distribution of the excited state can be considered as the image of the rotational distribution of the ground state.

The results of both methods coincide within experimental error, showing that the method of the gas temperature determination using molecular Fulcher- $\alpha$ (2-2)Q band of the hydrogen can be successfully used for the spectroscopic diagnostics of hydrogen containing HFEDLs. It is necessary to point out that there is a slight discrepancy between the rotational temperature and gas temperature at the applied current of $100 \mathrm{~mA}$; however this difference is just about $75 \mathrm{~K}$. 


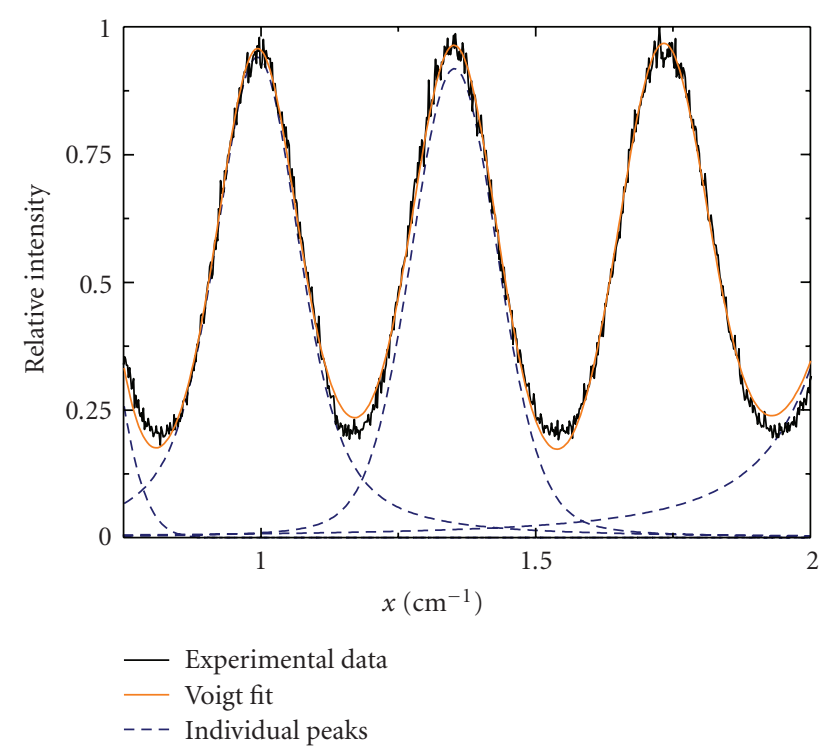

Figure 6: An example of experimental profile of helium emission line with wavelength $667.8 \mathrm{~nm}$ registered by means of Fabry-Perot interferometer at the applied current $i=100 \mathrm{~mA}$. The profile fitting with the Voigt function has been performed. Obtained gas temperature from the Doppler width was equal to $628 \pm 34 \mathrm{~K}$.

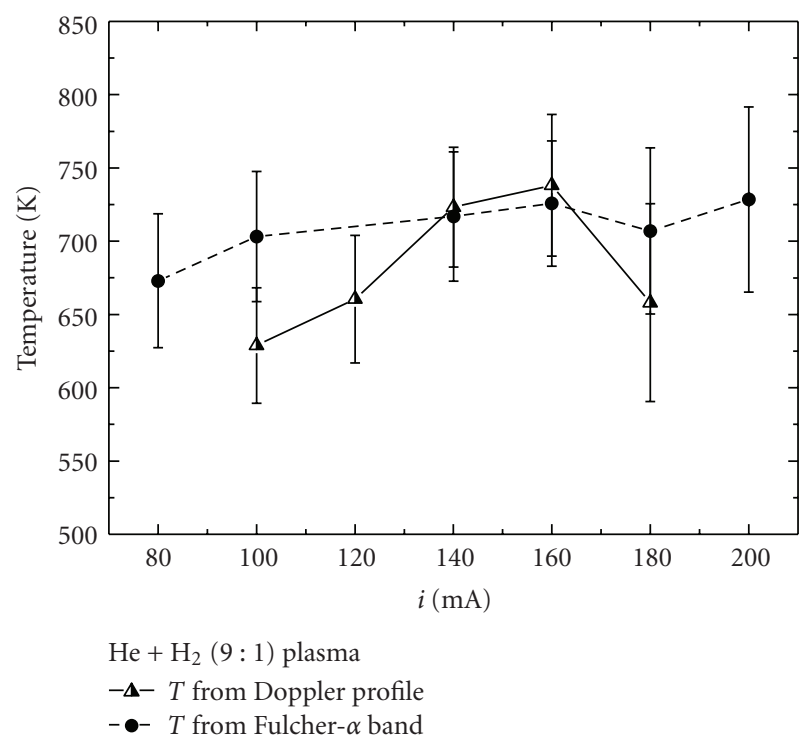

Figure 7: The temperature dependence on the applied current for the $\mathrm{He}+\mathrm{H}_{2}(9: 1) \mathrm{HFEDL}$. Temperature values obtained using $\mathrm{H}_{2}$ Fulcher- $\alpha$ (2-2)Q band are compared with the ones obtained from Doppler profile of helium emission line with wavelength $667.8 \mathrm{~nm}$.

4.2. Ar and $\mathrm{H}_{2}$ HFEDLs. Concerning $\mathrm{Ar}$ and $\mathrm{H}_{2}$ HFEDL, we used this lamp to estimate the gas temperature difference of argon HFEDL in the presence of hydrogen admixture and without it. As mentioned before, initially the HFELD was filled with 0.9 Torr $\mathrm{Ar}$ and 0.1 Torr $\mathrm{H}_{2}$. In Figure 8(a) one may see the emission spectra of the $\mathrm{Ar}+\mathrm{H}_{2}$ HFEDL recorded during the first measurement session, which consisted from

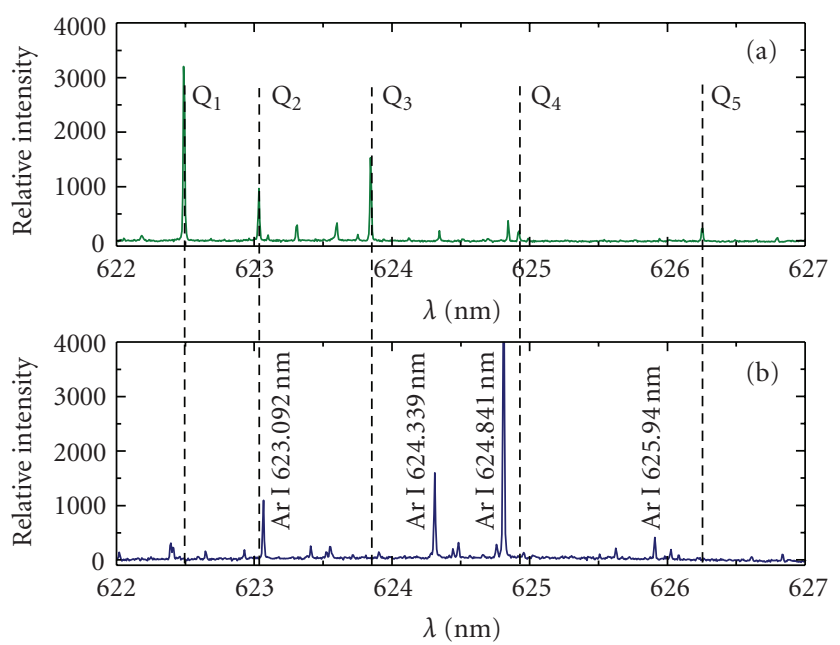

FIgURE 8: The emission spectra of the Ar HFEDL recorded at applied current $i=100 \mathrm{~mA}$, in the wavelength region 622$627 \mathrm{~nm}$ : (a) argon disharge with admixture of hydrogen (9:1) with hydrogen Fulcher- $\alpha$ (2-2)Q first five lines (first measurement session); (b) pure argon discharge without hydrogen (the control session).

several repeated measurements to assess experimental uncertainties. From this session we estimated the gas temperature using the rotational line spectrum of hydrogen. Later we operated the HFEDL so long until the hydrogen was diffused out from the lamp through the walls. The diffusion of the hydrogen was indicated registering the spectra in the same spectral region as before. The lamp spectra showed at the end that the Fulcher- $\alpha$ (2-2)Q band could not be detected anymore (Figure $8(\mathrm{~b})$ ). Due to the fact that hydrogen was lost from the lamp during measurements, also visually one could observe the changing color of the discharge. The gas temperature using the Doppler broadening of argon lines was estimated during the second measurement session.

In the Figure 9 the dependence of the estimated gas temperature in dependence of the applied current is shown for two cases described before. The Line 1 indicates the temperature dependence for argon and hydrogen discharge plasma, estimated from the $\mathrm{H}_{2}$ rotational lines of the (22)Q branch of the Fulcher- $\alpha$ band system during the first measurement session. Line 2 indicates the temperature for argon plasma after degassing of hydrogen, estimated from the Doppler broadening of Ar spectral line of $772.4 \mathrm{~nm}$ wavelength during the second measurement session.

The temperature of argon discharge with admixture of hydrogen was estimated about $620 \mathrm{~K}$ and changes not significantly by varying the applied current. Temperature estimated during second measurement session from Doppler broadening was changed from $620 \mathrm{~K}$ up to $1050 \mathrm{~K}$ (at applied current $180 \mathrm{~mA}$ ) because of the hydrogen loses, namely, the plasma temperature was increased. During the second session the plasma content was changed and at the end only argon plasma was present. 


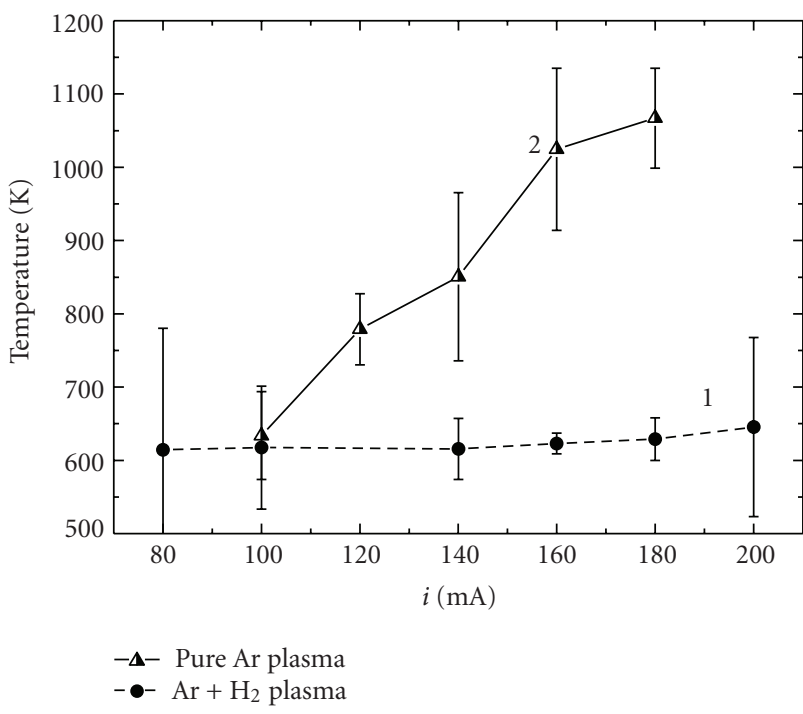

Figure 9: The temperature dependence on the applied current for the $\mathrm{Ar}$ and $\mathrm{H}_{2}$ containing HFEDL. Temperature values obtained using Fulcher- $\alpha$ (2-2)Q band are compared with ones obtained from Doppler profile of argon emission line with wavelength $772.4 \mathrm{~nm}$. Line 1 is temperature of $\mathrm{Ar}+\mathrm{H}_{2}(9: 1)$ plasma obtained from Fulcher- $\alpha$ (2-2)Q band; Line 2 is temperature of pure Ar plasma obtained from the Doppler profile.

The difference between the temperatures of plasma with $10 \%$ admixture of hydrogen and pure argon plasma increases with increasing applied current.

So the results, shown in Figure 9, allow concluding that the addition of about $10 \%$ hydrogen in argon plasma causes the significant decrease of the gas temperature. The observation of argon emission quenching by the addition of hydrogen has been reported elsewhere [11, 25-28]. For instance, in [25, Page 349] it was mentioned that the addition of hydrogen quenches the argon's excited states, and in one of our previous articles [11] we reported the observation of the similar phenomenon in the microwave $\mathrm{Ar}+\mathrm{H}_{2}$ plasma, where the increase of the hydrogen percentage in the $\mathrm{Ar}$ plasma decreased the population of resonant and metastable states of Ar.

In addition, authors of [26] report the temperature decrease with increasing the percentage of hydrogen in the argon plasma (DC plasma).

One of the possible mechanisms involved in the cooling of the plasma is quenching of excited argon atoms by hydrogen molecules in the ground state, leading to excitation with subsequent dissociation of $\mathrm{H}_{2}[27,28]$. Nevertheless it needs to be verified by detailed analysis of excitation and deexcitation processes in this kind of plasma.

\section{Conclusion}

From the results of our measurements we can conclude that it is possible to use relative intensities of the (2-2)Q branch of hydrogen Fulcher- $\alpha$ band for the gas temperature determination also in hydrogen containing high-frequency electrodeless lamps. The gas temperatures, obtained from Fulcher- $\alpha$ (2-2)Q band, are in good agreement with the ones obtained from the Doppler broadening of emission lines, but it is necessary to investigate further the applicability of this method for HFEDLs in the variety of experimental conditions.

Our experiments show that adding 10\% of hydrogen in the argon high-frequency electrodeless discharge lamps significantly reduces the plasma temperature comparing to temperature of the plasma without hydrogen.

\section{Acknowledgment}

The work was partly supported by Latvian Council of Science Grant no. 05.1866 .

\section{References}

[1] A. Ganeev, Z. Gavare, V. I. Khutorshikov, et al., "Highfrequency electrodeless discharge lamps for atomic absorption analysis," Spectrochimica Acta B, vol. 58, no. 5, pp. 879-889, 2003.

[2] G. Revalde, N. Denisova, Z. Gavare, and A. Skudra, "Diagnostics of capillary mercury-argon high-frequency electrodeless discharge using line shapes," Journal of Quantitative Spectroscopy and Radiative Transfer, vol. 94, no. 3-4, pp. 311-324, 2005.

[3] A. Skudra, G. Revalde, Z. Gavare, J. Silinsh, N. Zorina, and B. Polyakov, "Study of the high-frequency inductive coupled discharge plasma interaction with walls," Plasma Processes and Polymers, vol. 4, supplement 1, pp. S1026-S1029, 2007.

[4] A. Skudra, N. Zorina, Z. Gavare, M. Berzins, and D. Erts, "Light source inner surface changes depending on treatment," Physica Status Solidi C, vol. 5, pp. 915-917, 2008.

[5] A. Skudra and G. Revalde, "Mathematical modelling of the spectral line profiles in the high-frequency discharge," Journal of Quantitative Spectroscopy and Radiative Transfer, vol. 61, no. 6, pp. 717-728, 1999.

[6] G. Revalde, A. Skudra, N. Zorina, and S. Sholupov, "Investigation of $\mathrm{Hg}$ resonance $184.9 \mathrm{~nm}$ line profile in a lowpressure mercury-argon discharge," Journal of Quantitative Spectroscopy and Radiative Transfer, vol. 107, no. 1, pp. 164172, 2007.

[7] V. Schulz-von der Gathen and H. F. Döbele, "Critical comparison of emission spectroscopic determination of dissociation in hydrogen RF discharges," Plasma Chemistry and Plasma Processing, vol. 16, no. 4, pp. 461-486, 1996.

[8] S. A. Astashkevich, M. Käning, E. Käning, et al., "Radiative characteristics of $3 \mathrm{p} \sum, \Pi ; 3 \mathrm{~d} \Pi^{-}, \Delta^{-}$-states of $\mathrm{H}_{2}$ and determination of gas temperature of low pressure hydrogen containing plasmas," Journal of Quantitative Spectroscopy and Radiative Transfer, vol. 56, no. 5, pp. 725-751, 1996.

[9] T. Gans, V. Schulz-von der Gathen, and H. F. Döbele, "Time dependence of rotational state populations of excited hydrogen molecules in an RF excited plasma reactor," Plasma Sources Science and Technology, vol. 10, no. 1, pp. 17-23, 2001.

[10] M. Abdel-Rahman, T. Gans, V. Schulz-von der Gathen, and H. F. Döbele, "Space and time resolved rotational state populations and gas temperatures in an inductively coupled hydrogen RF discharge," Plasma Sources Science and Technology, vol. 14, no. 1, pp. 51-60, 2005. 
[11] Z. Gavare, D. Goett, A. V. Pipa, J. Roepcke, and A. Skudra, "Determination of the number densities of argon metastables in argon-hydrogen plasma by absorption and self-absorption methods," Plasma Sources Science and Technology, vol. 15, no. 3, pp. 391-395, 2006.

[12] L. Tomasini, A. Rousseau, G. Gousset, and P. Leprince, "Spectroscopic temperature measurements in a $\mathrm{H}_{2}$ microwave discharge," Journal of Physics D, vol. 29, no. 4, pp. 1006-1013, 1996.

[13] A. V. Pipa, On determination of the degree of dissociation of hydrogen in non-equilibrium plasmas by means of emission spectroscopy, Ph.D. thesis, University of Greifswald, Greifswald, Germany, 2004.

[14] R. K. Garg, T. N. Anderson, R. P. Lucht, T. S. Fisher, and J. P. Gore, "Gas temperature measurements in a microwave plasma by optical emission spectroscopy under single-wall carbon nanotube growth conditions," Journal of Physics D, vol. 41, no. 9, Article ID 095206, 2008.

[15] G. Lj. Majstorović, "Spectroscopic study of hydrogen rotational, vibrational and translational temperatures in a hollow cathode glow discharge," Journal of Physics: Conference Series, vol. 133, Article ID 012022, 2008.

[16] G. Lj. Majstorović, N. M. Šišović, and N. Konjević, "Rotational and vibrational temperatures of molecular hydrogen in a hollow cathode glow discharge," Plasma Sources Science and Technology, vol. 16, no. 4, pp. 750-756, 2007.

[17] S. Iordanova, "Spectroscopic temperature measurements in hydrogen inductively-driven plasmas at low pressures," Journal of Physics: Conference Series, vol. 113, no. 1, Article ID 012005, 2008.

[18] Yu. A. Lebedev and M. V. Mokeev, "Gas temperature in the plasma of a low-pressure electrode microwave discharge in hydrogen," Plasma Physics Reports, vol. 29, no. 3, pp. 226-230, 2003.

[19] Sh. Kado, D. Yamasaki, B. Xiao, et al., "On the anomalous characteristics in the $\mathrm{P}$ and $\mathrm{R}$ branches in a hydrogen fulcher band," Journal of Plasma and Fusion Research, vol. 7, pp. 54-58, 2006.

[20] B. Xiao, Sh. Kado, Sh. Kajita, and D. Yamasaki, "Rovibrational distribution determination of $\mathrm{H}_{2}$ in low temperature plasmas by Fulcher- $\alpha$ band spectroscopy," Plasma Physics and Controlled Fusion, vol. 46, no. 4, pp. 653-668, 2004.

[21] E. Surrey and B. Crowley, "Spectroscopic measurement of gas temperature in the neutralizer of the JET neutral beam injection system," Plasma Physics and Controlled Fusion, vol. 45, no. 7, pp. 1209-1226, 2003.

[22] H. M. Crosswhite, Ed., The Hydrogen Molecule Wavelength Tables of Gerhard Heinrich Dieke, Wiley-Interscience, New York, NY, USA, 1972.

[23] S. A. Alexander and R. L. Coldwell, "Spectroscopic constants of $\mathrm{H}_{2}$ using Monte Carlo methods," International Journal of Quantum Chemistry, vol. 100, no. 6, pp. 851-857, 2004.

[24] I. Dabrowski, "The Lyman and Werner bands of $\mathrm{H}_{2}$," Canadian Journal of Physics, vol. 62, pp. 1639-1664, 1984.

[25] H. Yasuda, Luminous Chemical Vapor Deposition and Interface Engineering, Surfactant Science, CRC Press, Boca Raton, Fla, USA, 2004.

[26] I. Ishii, T. G. Beuthe, J. S. Chang, et al., "The influence of hydrogen gas mixtures on the argon thermal plasma temperature," Research Reports of the Faculty of Engineering, Tokyo Denki University, vol. 46, pp. 11-22, 1998.
[27] A. Bogaerts, "Hydrogen addition to an argon glow discharge: a numerical simulation," Journal of Analytical Atomic Spectrometry, vol. 17, no. 8, pp. 768-779, 2002.

[28] A. Bogaerts, Z. Chen, and R. Gijbels, "Glow discharge modelling: from basic understanding towards applications," Surface and Interface Analysis, vol. 35, no. 7, pp. 593-603, 2003. 


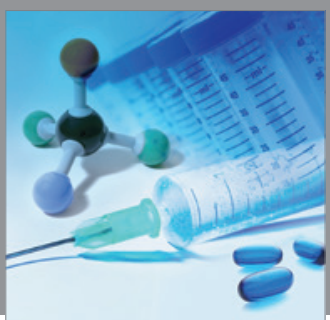

International Journal of

Medicinal Chemistry

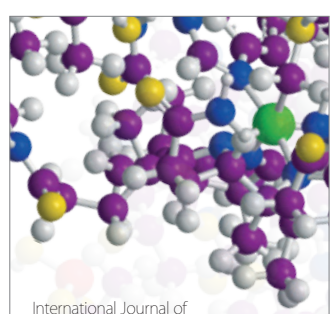

Carbohydrate Chemistry

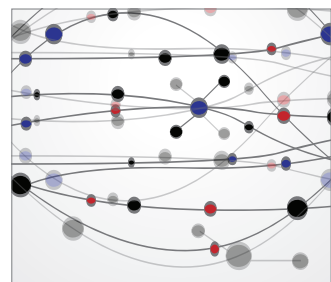

The Scientific World Journal
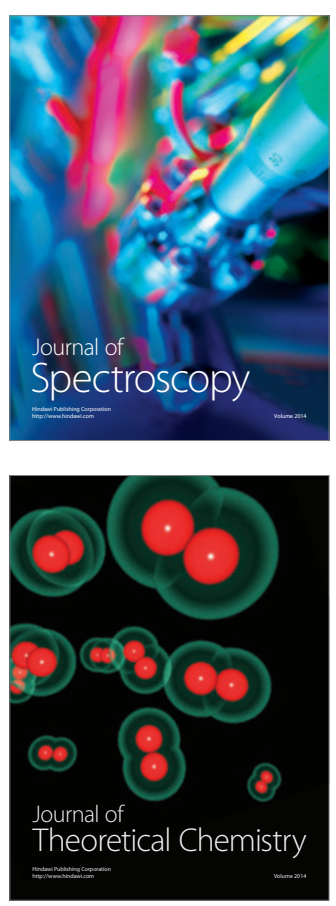
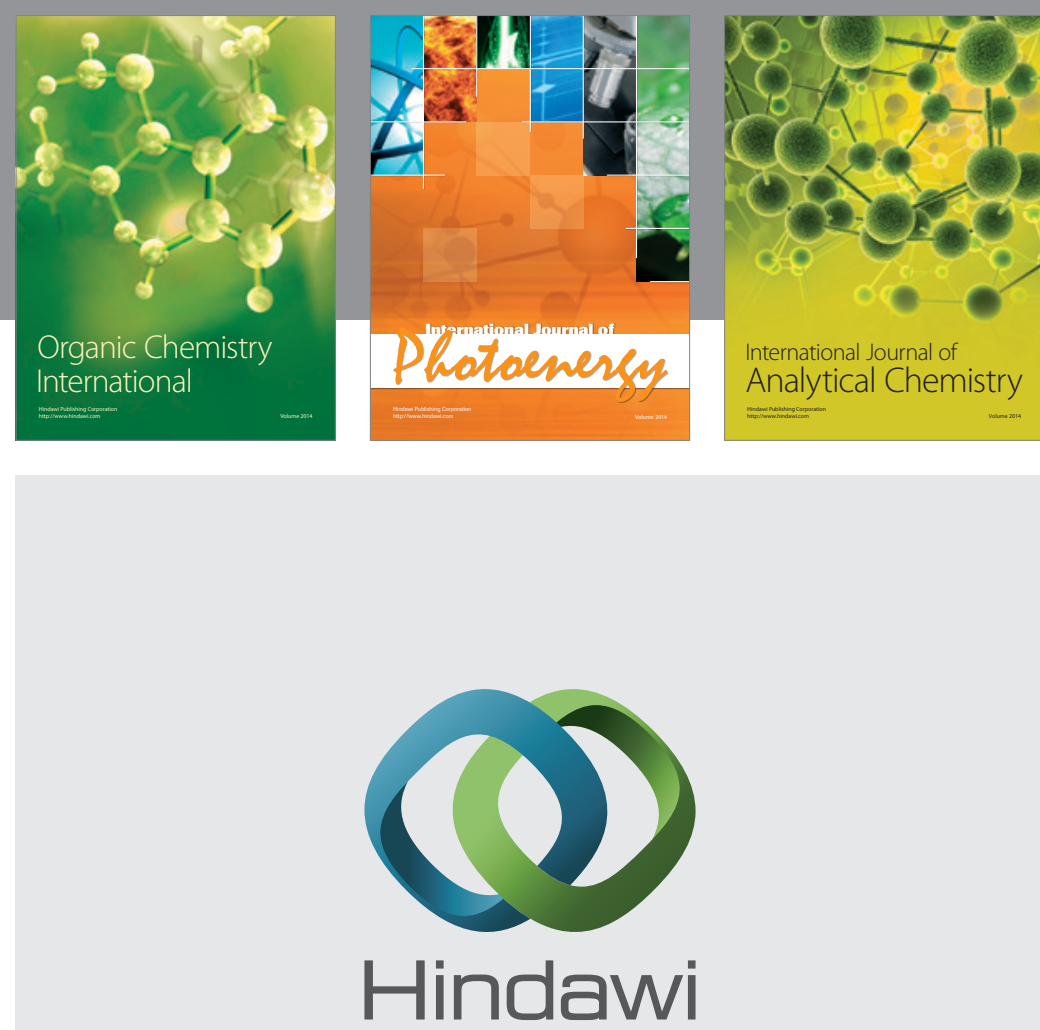

Submit your manuscripts at

http://www.hindawi.com
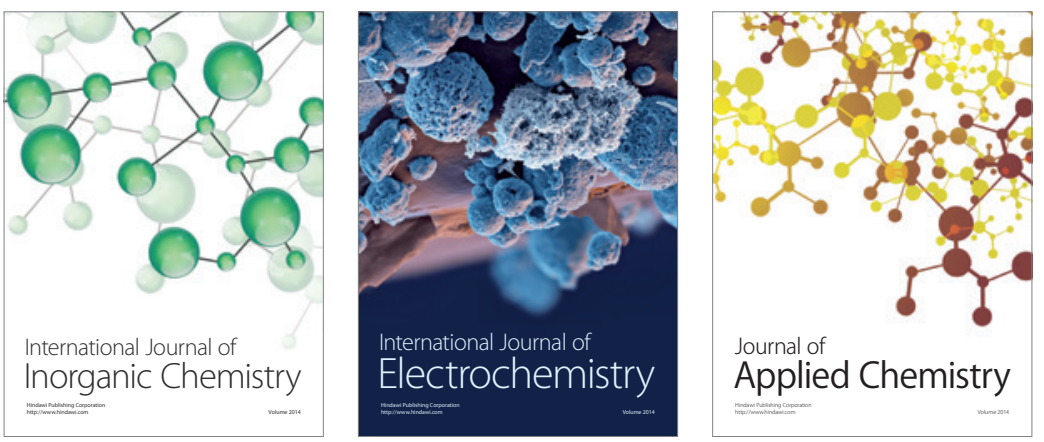

Journal of

Applied Chemistry
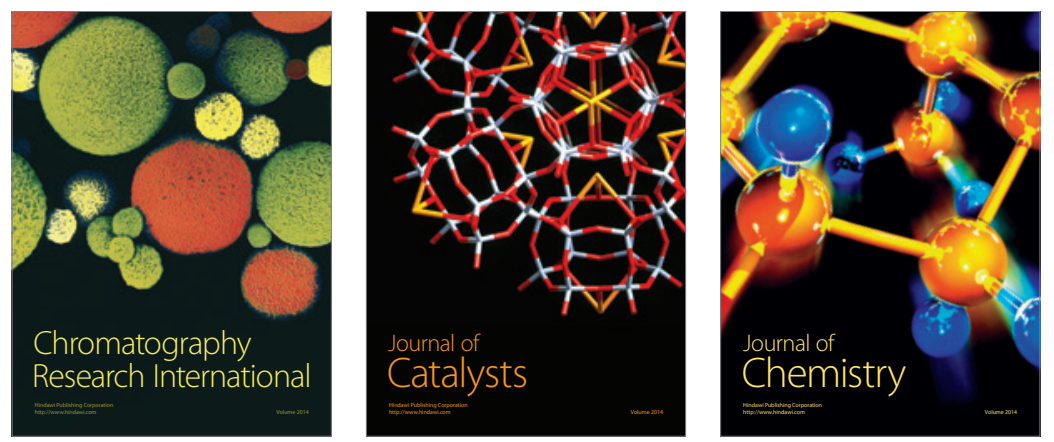
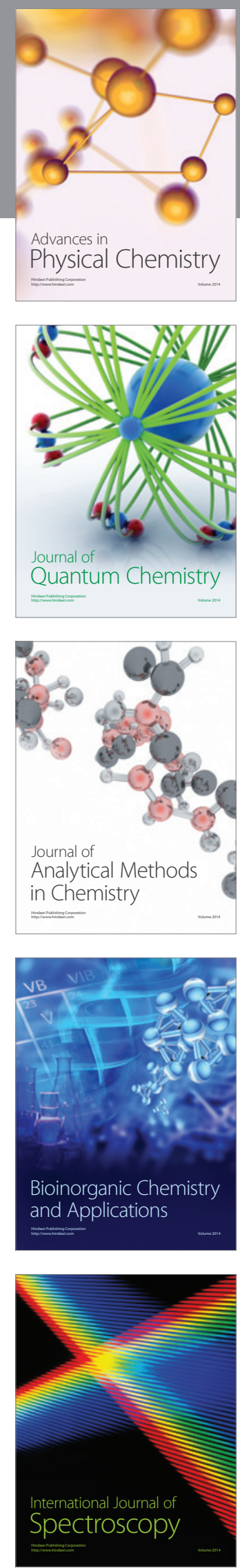